\title{
LETTERS
}

\section{Understanding the application of diagnostic criteria for multiple sclerosis helps prevent misdiagnosis}

I would like to highlight several considerations regarding application of the 2017 McDonald criteria for multiple sclerosis (MS) in the case reported by Nasser and colleagues $^{1}$ in CMAJ. The authors stated that MS is "an atypical cause" of this patient's lateral pontine syndrome (not lateral medullary syndrome, as they assert); if this were the case then caution should have been advised when applying the 2017 McDonald criteria, which are intended to be used primarily in patients with typical clinically isolated syndromes. ${ }^{2}$ A subacute focal brainstem presentation, however, is a typical clinically isolated syndrome $^{2}$ and use of the 2017 McDonald criteria is therefore appropriate (the patient age of onset may be atypical for MS, but the neurologic presentation is not).

Regarding application of these criteria, disease dissemination both in time and space is required for the diagnosis of MS. ${ }^{2}$ In this type of patient with 1 or more characteristic lesions in 2 or more of 4 areas in the central nervous system (i.e., periventricular, infratentorial brain and spinal cord), dissemination in space criterion has been met. ${ }^{2}$ However, the authors reported that their case of MS was "fulfilling McDonald criteria with the patient having 1 "attack" and objective clinical evidence of an indicative lesion on [magnetic resonance imaging] MRI in 2 areas of the central nervous system." This explanation does not describe how the patient met the criterion for dissemination in time. To meet this criterion, a patient must have multiple clinical attacks, the simultaneous presence of enhancing and nonenhancing lesions or new lesions on follow-up MRI with reference to a baseline scan, ${ }^{2}$ none of which are clearly stated in this article. The patient had only 1 clinical attack, 2 enhancing lesions without mention of simultaneous nonenhancing lesions and no reported new lesion compared with previous imaging (the enhancing periventricular lesion is not explicitly stated as a new lesion in comparison to the noncontrast MRI performed on presentation that showed "chronic microangiopathic ischemic changes or demyelination" in the deep white matter; the cervical cord was imaged only once, meaning the observed lesion is also not clearly new from the time of presentation).

Without further description or depiction of the lesions in the deep white matter it is difficult to gauge which of these may be demyelinating in nature, which would aid in determining whether simultaneous enhancing and nonenhancing lesions or new lesions were present at the time of contrast-enhanced MRI. The presence of oligoclonal bands specific to cerebrospinal fluid may substitute for the dissemination in time criterion ${ }^{2}$ but were absent in this case.

To clarify, I do not necessarily disagree with the diagnosis of MS in this patient. However, in teaching cases such as this it is imperative that the reasoning behind why a patient fulfills the 2017 McDonald criteria is clearly outlined; otherwise, this may lead to incorrect application of these criteria in future cases and potential misdiagnoses of MS.

\section{Adrian Budhram MD}

Autoimmune neurology fellow, Mayo

Clinic Hospital, Rochester, Minn.

Cite as: CMAJ 2020 August 4;192:E894. doi: $10.1503 / \mathrm{cmaj} .76124$

\section{References}

1. Nasser L, Deshauer S, Caners K, et al. A 67-yearold man with facial droop, ataxia and vertigo. CMAJ 2020;192:E626-9.

2. Thompson AJ, Banwell BL, Barkhof F, et al. Diagnosis of multiple sclerosis: 2017 revisions of the McDonald criteria. Lancet Neurol 2018;17:162-73.

Competing interests: None declared. 